\title{
Estudo de Perfusão Miocárdica e Cineangiocoronariografia em pacientes com Lúpus Eritematoso Sistêmico ${ }^{(*)}$
}

\author{
Myocardial Perfusion Study and Coronary Artery Angiography in \\ Systemic Lupus Erythematosus Patients
}

\author{
Elaine Marcelina Claudio Sella ${ }^{(1)}$ e Emilia Inoue Sato ${ }^{(2)}$
}

\section{RESUMO}

Objetivo: avaliar as artérias coronárias por meio da cineangiocoronariografia de pacientes com lúpus eritematoso sistêmico (LES) e anormalidades de perfusão miocárdica. Métodos: participaram do estudo 90 pacientes do sexo feminino, idades entre 18 e 55 anos, com diagnóstico de LES há mais de cinco anos, sem antecedentes pessoais de doença arterial coronária (DAC) manifesta e em uso atual ou pregresso de corticosteróide por, pelo menos, um ano. A cintilografia cardíaca, com aquisição de imagens tomográficas utilizando Tecnécio $99 \mathrm{~m}$-sestamibi, foi executada nas fases de repouso e de estresse induzido por dipiridamol. Os fatores de risco (FR) considerados foram os tradicionais para DAC e os relacionados ao LES. As pacientes com alterações cintilográficas foram convidadas a submeterem-se à cineangiocoronariografia. Resultados: as pacientes apresentaram média de idade de $38 \pm 10$ anos, diagnóstico de LES há $128 \pm 59$ meses, número de critérios do American College of Rheumatology (ACR) $7 \pm 1$, escores do Disease Activity Index for Systemic Lupus Erythematosus (SLEDAI) $6 \pm 5$ e do Systemic Lupus International Collaborating Clinics/ACR Damage Index for SLE (SLICC/ACR-DI) $2 \pm 2$. Anormalidades de perfusão miocárdica foram observadas em 33\% das pacientes. A análise de regressão logística considerando-se os FR-DAC tradicionais e as variáveis relacionadas ao LES foi realizada com 48 pacientes em seguimento regular. Vasculite atual foi associada à anormalidade de perfusão miocárdica. Não houve associação entre outras manifestações clínicas e/ou sorológicas de LES, dose cumulativa ou tempo de uso de prednisona e o resultado da cintilografia miocárdica. Vinte e uma pacientes com alterações cintilográficas foram submetidas à cineangiocoronariografia. Placas ateroscleróticas em artérias coronárias foram observadas em 8 pacientes $(38 \%)$. O subgrupo das pacientes com cateterismo

\section{ABSTRACT}

Objective: To evaluate coronary artery findings using coronary artery angiography in systemic lupus erythematosus (SLE) patients with myocardial perfusion abnormalities. Methods: Ninety female SLE patients, ages from 18 to 55 years old, more than 5 years of disease duration, without current or previous confirmed coronary artery disease (CAD), and who were either in use or had used steroid treatment for at least 1 year were evaluated. Myocardial perfusion scintigraphy was performed using a singlephoton emission computed tomography employing Technetium 99m-sestamibi. Images were captured at resting and after dipyridamole-induced stress. The CAD risk factors (RF) considered were the traditional ones in general population and SLE-related factors. All patients who had abnormal findings on myocardial perfusion tests were invited to undergo coronary angiography. Results: The mean age was $38 \pm 10$ years old, with mean disease duration of $128 \pm 59$ months, American College of Rheumatology (ACR) number $7 \pm 1$, Disease Activity Index for SLE (SLE-DAI) score $6 \pm 5$ and Systemic Lupus International Collaborating Clinics/ACR Damage Index for SLE (SLICC/ACR-DI) score $2 \pm 2$. Thirty-three percent of patients presented myocardial perfusion scintigraphy abnormalities. Regression logistic analysis was performed considering the traditional CAD-RF and SLE-related variables in 48 SLE patients on regular follow-up. Current vasculitis was associated with abnormal myocardial perfusion. There was no association between other clinical and/or serologic features, cumulative dose or duration of prednisone use and myocardial perfusion scintigraphy results. Twenty-one patients with abnormal myocardial scintigraphy agreed to undergo coronary angiography. Atherosclerotic plaques were identified by

\footnotetext{
* Este estudo teve apoio financeiro da Fundação de Amparo à Pesquisa do Estado de São Paulo (Fapesp) - processo 98/1 1794-6 - e foi parcialmente publicado no Arthritis \& Rheumatism 48 (1 1): 3168-75, 2003. Recebido em 04/03/2004. Aprovado, após revisão, em 12/04/2004.

1. Mestre em reumatologia - Disciplina de Reumatologia - pela Universidade Federal de São Paulo (Unifesp)/Escola Paulista de Medicina (EPM).

2. Professora titular da Disciplina de Reumatologia da Unifesp/EPM.
}

Endereşo para correspondência: Elaine Marcelina Claudio Sella. Disciplina de Reumatologia da Universidade Federal de São Paulo/Escola Paulista de Medicina (Unifesp/EPM) Rua Botucatu, 740, 30 andar, Vila Clementino, CEP 04023-062, São Paulo, SP. E-mail: emiliasato@reumato.epm.br 
cardíaco anormal apresentou maior número de FR clássicos para DAC $(p=0,006)$. Hipertensão arterial sistêmica, menopausa e hipertrigliceridemia foram os FR-DAC associados à presença de lesões coronárias ( $\mathrm{p}=0,046 ; 0,024$ e 0,043, respectivamente). Número de critérios do ACR e escores do SLE-DAI e do SLICC/ ACR-DI também foram significativamente maiores no subgrupo de pacientes com cateterismo alterado $(p=0,018 ; 0,010$ e 0,003, respectivamente). Conclusões: vasculite atual foi importante variável associada à anormalidade cintilográfica. Este estudo sugere que a cintilografia miocárdica pode ser usada como rastreamento de DAC em pacientes com LES e risco aumentado para doença cardiovascular, mesmo na ausência de sintomas de isquemia cardíaca. Pacientes com anormalidades de perfusão miocárdica e, pelo menos, 4 FR-DAC têm maior chance de apresentar estenoses coronárias à cineangiocoronariografia.

Palavras-chave: lúpus eritematoso sistêmico, doença arterial coronária, fatores de risco, cintilografia miocárdica, cineangiocoronariografia.

\section{INTRODUÇÃO}

A doença arterial coronária (DAC), resultante de aterosclerose prematura no lúpus eritematoso sistêmico (LES), tem sido amplamente estudada. Há, no entanto, aspectos não elucidados, como a possibilidade de um paralelo entre a patogênese de doença reumatológica imunologicamente mediada como o LES e a aterosclerose na população geral $^{(1)}$.

Estudos de aterogênese sugerem que a progressão da placa aterosclerótica na população geral seja determinada por processo relacionado às desordens inflamatórias ${ }^{(2-5)}$. Hipoteticamente, analisando-se a similaridade entre o LES como doença marcada por processo inflamatório crônico e a característica inflamatória dos componentes celulares da placa aterosclerótica, poder-se-ia inferir que muitas placas, em pacientes com LES, possam ser vulneráveis à ruptura, propiciando a ocorrência de eventos trombóticos ${ }^{(6)}$.

Investigações epidemiológicas têm sido fundamentais para o estabelecimento dos fatores de risco coronário tradicionais que contribuem para DAC em pacientes com $\operatorname{LES}^{(7-12)}$. Os fatores de risco coronário tradicionais em pacientes jovens com LES parecem ser similares aos clássicos fatores de risco da população geral ${ }^{(13)}$.

A comparação de subgrupos de pacientes com e sem eventos cardiovasculares em estudos anteriores apontou que idade mais avançada na época do diagnóstico de LES, longo angiography in eight patients (38\%). Abnormal angiography subgroup presented higher number of traditional CAD-RF $(p=0.006)$. Arterial hypertension, postmenopausal status and higher triglyceride level showed a significant association with abnormal angiography ( $p=0.046,0.024$ and 0.043, respectively). The number of ACR criteria, SLE-DAI and SLICC/ACR-DI scores were also higher in the sub-group with coronary abnormalities ( $p=0.018,0.010$ and 0.003, respectively). Conclusions: Current vasculitis was an important variable associated with abnormal myocardial scintigraphy. This study suggests that myocardial scintigraphy can be used as a screening test for CAD in SLE patients with high-risk of cardiovascular disease, even in the absence of ischemic heart symptoms. Patients with abnormal myocardial perfusion results and at least four CAD-RF had higher risk of presenting coronary stenosis on coronary angiography.

Keywords: systemic lupus erythematosus, coronary artery disease, risk factors, myocardial scintigraphy, coronary angiography.

tempo de diagnóstico da doença e longo período de corticoterapia foram os fatores significativamente associados com clínica de $\operatorname{DAC}^{(8,9,14)}$.

$\mathrm{Na}$ população geral, a cineangiocoronariografia é considerada técnica diagnóstica padrão-ouro e de valor inquestionável na avaliação de vasos coronários. A abordagem inicial de pacientes com LES com "alto risco" para DAC pela cineangiocoronariografia seria, no entanto, muito invasiva e não-ética, motivos pelos quais não dispomos de estudos angiográficos na doença. Nos últimos anos, vários métodos não-invasivos têm sido empregados para o rastreamento de DAC nesses pacientes, mas não há consenso sobre o melhor método diagnóstico para a avaliação de possível DAC em pacientes com LES.

Anormalidades de perfusão miocárdica foram relatadas em $16 \%$ a $82 \%$ dos pacientes com LES ${ }^{(15-19)}$. A cintilografia cardíaca não é um teste diagnóstico de insuficiência coronária, porém a alteração cintilográfica resultante da intervenção farmacológica com dipiridamol sugere a sua existência, pela hipoperfusão devida à isquemia ou desproporção de fluxo ${ }^{(20)}$.

Não há na literatura, até o momento, trabalhos com cineangiocoronariografia e estudo de perfusão cardíaca em pacientes com LES. Este estudo tem por objetivo principal avaliar as artérias coronárias mediante a cineangiocoronariografia de pacientes com LES e anormalidades de perfusão miocárdica observadas por cintilografia cardíaca com Tecnécio-99m ( $\left.{ }^{99 m} \mathrm{Tc}\right)$ - sestamibi. 


\section{PACIENTES E MÉTODOS}

No período compreendido entre setembro de 1998 e setembro de 2000 foram convidadas a participar do estudo pacientes com diagnóstico de LES em seguimento no Ambulatório de Doenças do Tecido Conjuntivo da Disciplina de Reumatologia do Hospital São Paulo Universidade Federal de São Paulo/Escola Paulista de Medicina (Unifesp/EPM).

A seleção de pacientes obedeceu aos seguintes critérios de inclusão: presença de quatro ou mais critérios para a classificação de LES, de acordo com a proposição do American College of Rheumatology - ACR - ${ }^{(21,22)}$, diagnóstico há mais de cinco anos, sexo feminino, idades entre 18 e 55 anos e uso atual ou pregresso de corticosteróide por período de, no mínimo, um ano.

Os critérios de exclusão foram definidos conforme as contra-indicações ao protocolo de estresse farmacológico com dipiridamol ${ }^{(23)}$. As pacientes com DAC manifesta - infarto agudo do miocárdio (IAM) atual ou pregresso definidos, angioplastia ou cirurgia de revascularização miocárdica prévias -, gestantes e lactantes foram excluídas do estudo.

$\mathrm{O}$ projeto de pesquisa foi previamente aprovado pela Comissão de Ética em Pesquisa Médica da Instituição. Todas as participantes foram informadas sobre o protocolo de pesquisa e assinaram o termo de consentimento pósinformação.

A revisão dos prontuários foi realizada com o objetivo de se obter informações a respeito do tempo de diagnóstico do LES, critérios diagnósticos e outras manifestações clínicas da doença (outras vasculites incluíram úlceras não mucosas ou digitais, vasculites viscerais e do sistema nervoso central comprovadas por exames subsidiários), dados laboratoriais gerais e imunológicos, lesões irreversíveis em órgãos e/ou sistemas, tempo de uso de agentes antimaláricos e tempo de uso e dose cumulativa de prednisona.

Para o cálculo do tempo de uso de agentes antimaláricos e tempo de uso e dose cumulativa de prednisona foram consideradas apenas as pacientes que tiveram diagnóstico de LES na Instituição e permaneceram, desde então, em acompanhamento clínico regular a intervalos não superiores a quatro meses.

As pacientes foram submetidas ao exame físico geral e à coleta de sangue e urina para a realização dos exames laboratoriais. Todas foram avaliadas quanto à atividade da doença através do Disease Activity Index for Systemic Lupus Erythematosus (SLE-DAI) ${ }^{(24)}$ e quanto aos danos permanentes através do Systemic Lupus International Collaborating Clinics/ACR Damage Index for SLE (SLICC/ ACR-DI) ${ }^{(25,26)}$, por ocasião do estudo.

A glicemia e o perfil lipídico (colesterol total, HDL-c, LDL-c, VLDL-c e triglicérides) foram solicitados para todas as participantes do estudo. A coleta de sangue, próxima à realização da cintilografia cardíaca, foi feita após 12 horas de jejum. As pacientes foram orientadas a evitar abusos alimentares e/ou alcoólicos no dia anterior à coleta de sangue. Os índices de Castelli I e II foram obtidos pelos cálculos colesterol total/ HDL-c e LDL-c/HDL-c, respectivamente ${ }^{(27,28)}$.

Os fatores de risco coronário tradicionais considerados foram: hipertensão arterial sistêmica (HAS), diabetes mellitus, HDL-c $<35 \mathrm{mg} / \mathrm{dL}$, LDL-c $\geq 130 \mathrm{mg} / \mathrm{dL}$, triglicérides $\geq 200 \mathrm{mg} / \mathrm{dL}$, menopausa, tabagismo, obesidade e história familiar de DAC prematura ${ }^{(27,29,30)}$. A dor torácica foi classificada em angina pectoris, dor atípica e dor nãosugestiva de causa cardíaca, de acordo com o American College of Cardiology ${ }^{(31)}$.

$\mathrm{O}$ estudo de perfusão miocárdica com ${ }^{99 \mathrm{~m}} \mathrm{~T} \mathrm{c}$ - sestamibi foi executado em duas fases: de repouso e de estresse induzido farmacologicamente com dipiridamol, segundo protocolo padrão ${ }^{(23,32,33)}$ descrito em estudo por nós realizado ${ }^{(13)}$. O exame cintilográfico definido como normal foi aquele em que não se detectaram áreas de hipoconcentração do radiofármaco no ventrículo esquerdo nas duas séries de imagens. $O$ estudo cintilográfico anormal foi interpretado como defeito de perfusão reversível (sugestivo de isquemia miocárdica), fixo (sugestivo de fibrose) ou combinado (defeito reversível associado ao fixo).

As imagens cintilográficas foram avaliadas independentemente por três observadores, que desconheciam os dados clínicos e/ou fatores de risco coronário das pacientes examinadas. Caso houvesse discordância de laudo de pelo menos um dos observadores, o exame era reavaliado em reunião conjunta com os três profissionais, para a emissão do laudo final de consenso.

As pacientes com anormalidades cintilográficas foram encaminhadas para estudo angiográfico no setor de Cardiologia Invasiva da Instituição. Estas pacientes foram regularmente acompanhadas no ambulatório da Disciplina de Cardiologia durante todo o período do estudo.

\section{ANÁLISE ESTATÍSTICA}

O teste de qui-quadrado e o teste exato de Fisher foram utilizados para verificar a relação de dependência entre as variáveis categóricas. O teste de Mann-Whitney foi empre- 
gado para comparar os subgrupos de pacientes com relação às variáveis quantitativas ${ }^{(34)}$. Foram considerados estatisticamente significativos valores de $\mathrm{p}<0,05$.

A análise de regressão logística foi adotada para estudar a influência das diferentes variáveis na determinação do resultado do estudo de perfusão miocárdica. Anormalidade de cintilografia cardíaca foi considerada variável dependente e as variáveis independentes analisadas foram os fatores de risco coronário tradicionais e os relacionados ao LES. No modelo final da análise permaneceram as variáveis significantes que explicam as alterações do estudo cintilográfico. Os resultados foram expressos em odds ratio (OR), indicando a chance de se encontrar anormalidades cintilográfica em pacientes que tenham sido expostas às variáveis independentes analisadas ${ }^{(35)}$.

\section{RESULTADOS}

\section{DADOS DEMOGRÁFICOS E CLÍNICOS}

Participaram deste estudo 90 pacientes, 60\% de cor branca, com idades de $38 \pm 10$ anos (variação de 20 a 55 anos). $\mathrm{O}$ tempo de diagnóstico do LES foi $128 \pm 59$ meses (61 a 312 meses). A idade das pacientes na época do diagnóstico da doença foi $27 \pm 9$ anos (12 a 45 anos). O número de critérios do ACR foi $7 \pm 1$, os escores do SLE-DAI de $6 \pm 5$ (0 a 18) e do SLICC/ACR-DI de $2 \pm 2$ (0 a 6).

A vasculite digital foi descrita em prontuários de 46 pacientes (51\%), vasculite de mucosa oral ou nasal em 34 $(38 \%)$, outras vasculites em $16(18 \%)$, trombose venosa em $6(7 \%)$ e trombose arterial em $3(3 \%)$. No momento do estudo, vasculite foi observada em 15 pacientes (17\%), sendo vasculite digital em 15\% e ulceração em membros inferiores em 2\%. Na revisão dos prontuários, anticorpos anticardiolipina (aCL) foram positivos em 34 pacientes (38\%), e na ocasião do estudo, em 27 pacientes (30\%).

Trinta e três por cento das pacientes apresentaram dor de causa não cardíaca, 11\% dor atípica e 9\% angina pectoris por ocasião do estudo.

\section{ESTUDO DE PERFUSÃO MIOCÁRDICA}

Anormalidades de perfusão miocárdica foram observadas em 30 pacientes (33\%). Cinqüenta e oito por cento do total dos defeitos cintilográficos foram considerados como reversíveis, $18 \%$ fixos e $24 \%$ combinados (defeitos reversíveis associados aos fixos). A parede cardíaca mais freqüentemente afetada foi a anterior do ventrículo esquerdo.

Não houve associação estatisticamente significativa entre manifestações articulares, musculares, dermatológicas, hematológicas, renais, neurológicas, vasculares, pulmonares, cardíacas, de serosas ou imunológicas observadas ao longo da evolução da doença e anormalidades cintilográficas. Não observamos associação estatisticamente significativa entre positividade para anticorpos aCL (atual ou pregressa) e alteração da perfusão miocárdica. Trinta por cento das pacientes com exame cintilográfico anormal apresentavam vasculite digital na época do estudo de perfusão miocárdica.

Sete das 8 pacientes com angina pectoris (88\%) e 23 das 81 sem angina (28\%) apresentaram cintilografia miocárdica anormal ( $\mathrm{p}=0,003)$. Quarenta e três por cento das pacientes com cintilografia miocárdica anormal e 12\% daquelas com exame normal tinham pelo menos quatro fatores de risco para DAC $(\mathrm{p}=0,002)$.

AVALIAÇÃO DA INFLUÊNCIA DOS DIFERENTES FATORES DE RISCO RELACIONADOS AO LES NO ESTUDO DE PERFUSÃO MIOCÁRDICA

Quarenta e oito pacientes tiveram diagnóstico e seguimento clínico contínuo em nosso serviço. Vinte e nove (60\%) apresentaram cintilografia miocárdica normal e 19 (40\%) alterada. Embora o tempo de uso e a dose total de prednisona fossem maiores no subgrupo de pacientes com alterações de perfusão miocárdica, não foi encontrada diferença estatisticamente significativa entre os subgrupos de pacientes com cintilografia normal e anormal (Tabela 1).

TABELA 1

Medidas de tendÊnCIA CENTRAL E DE DisPersão do TEMPO DE USO E DOSE CUMULATIVA APROXIMADA DE PREDNISONA DAS 48 PACIENTES COM LES EM SEGUIMENTO CONTÍNUO DESDE O DIAGNÓSTICO DA DOENÇA

\begin{tabular}{lcccc}
\hline Variáveis & 48 & 29 & 19 & $\mathbf{P}$ \\
& LES & MIBI-NL & MIBI-ANL & \\
\hline $\begin{array}{l}\text { Corticoterapia } \\
\text { (meses) }\end{array}$ & & & & \\
MD \pm DP & $98 \pm 50$ & $89 \pm 36$ & $112 \pm 65$ & \\
Mediana & 88 & 81 & 109 & 0,292 \\
Mín./máx. & $27 / 272$ & $35 / 178$ & $27 / 272$ &
\end{tabular}

Dose cumulativa

(g)

$\begin{array}{lcccc}\text { MD } \pm \mathrm{DP} & 54 \pm 28 & 49 \pm 25 & 61 \pm 32 & \\ \text { Mediana } & 45 & 42 & 56 & 0,161 \\ \text { Mín./máx. } & 12 / 137 & 12 / 109 & 15 / 137 & \end{array}$

Teste de Mann-Whitney

$M D \pm D P$ - Média \pm desvio-padrão; MIBI ANL - Cintilografia miocárdica anormal; MIBI NL - Cintilografia miocárdica normal; Mín./máx. - Valores mínimo e máximo das variáveis; $P$ - Nível descritivo 
Quarenta e quatro pacientes estavam em uso ou haviam usado agentes antimaláricos. Não foi observada diferença estatística significativa entre médias, desvios-padrão e medianas do tempo de uso de antimaláricos entre os subgrupos com cintilografia normal e anormal.

A análise de regressão logística, realizada com as 48 pacientes, considerou as seguintes variáveis: idade da paciente na ocasião do exame, tempo de diagnóstico do LES, idade da paciente na época do diagnóstico da doença, escores do SLE-DAI e do SLICC/ACR-DI, vasculite digital e outras vasculites (revisão de prontuários), tempo de uso e dose cumulativa de prednisona, vasculite atual, anticorpos aCL (atuais) e número de fatores de risco tradicionais para DAC. Vasculite atual foi a variável associada à anormalidade cintilográfica $-\mathrm{p}=0,015$, $\mathrm{OR}=23,49$ e intervalo de confiança $(\mathrm{IC})=1,83-301,02$.

\section{ESTUDO DE PERFUSÃO MIOCÁRDICA E CINEANGIOCORONARIOGRAFIA}

Das 30 pacientes com anormalidades cintilográficas, 21 (42 \pm 9 anos e $62 \%$ da cor branca) concordaram em submeterem-se à cineangiocoronariografia. Oito (38\%) destas pacientes apresentaram estenose das artérias coronárias por placas ateroscleróticas. Em 87\% dos casos o comprometimento foi da artéria descendente anterior.

O subgrupo das pacientes com lesões em artérias coronárias apresentou número de fatores de risco para DAC significativamente maior do que o subgrupo sem lesões coronárias $(4,5 \pm 0,8$ e mediana 4 versus $2,5 \pm 1,9$ e mediana $2 ; \mathrm{p}=0,006)$. Oito pacientes $(100 \%)$ com cateterismo cardíaco alterado e 4 das 13 pacientes (31\%) com cateterismo normal apresentaram, pelo menos, quatro fatores de risco para DAC $(\mathrm{p}=0,005)$.

HAS esteve presente em todas as pacientes com lesões coronárias e em $54 \%$ das pacientes com coronárias normais $(p=0,046)$. Menopausa foi observada em $87 \%$ das pacientes com alteração coronária e em 31\% das pacientes com cateterismo normal $(p=0,024)$. Sete pacientes com angina pectoris apresentaram anormalidades cintilográficas e todas foram submetidas à cineangiocoronariografia. Três delas (43\%) apresentaram cateterismo normal e 4 (57\%) tinham angiografia alterada $(p>0,05)$. As variáveis triglicérides sérico e índices de Castelli I e II foram significativamente maiores no subgrupo das pacientes com cateterismo cardíaco alterado (Tabela 2).

Com relação aos fatores de risco relacionados ao LES, as variáveis número de critérios do ACR e escores do SLE-DAI e do SLICC/ACR-DI foram significativamente maiores no subgrupo das pacientes com lesões coronárias (Tabela 3).
TABELA 2

MEdidAS DE TENDÊNCIA CENTRAL E DE DISPERSÃO DOS FATORES DE RISCO PARA DAC DAS 21 PACIENTES COM LES SUBMETIDAS À CINEANGIOCORONARIOGR AFIA

\begin{tabular}{|c|c|c|c|c|}
\hline Variáveis & $\begin{array}{l}21 \\
\text { LES }\end{array}$ & $\begin{array}{c}13 \\
\text { CATE-NL }\end{array}$ & $\begin{array}{c}8 \\
\text { CATE-ANL }\end{array}$ & $\mathbf{P}$ \\
\hline \multicolumn{5}{|l|}{$\begin{array}{l}\text { Idade atual } \\
\text { (anos) }\end{array}$} \\
\hline$M D \pm D P$ & $42 \pm 9$ & $39 \pm 10$ & $47 \pm 4$ & \\
\hline $\begin{array}{l}\text { Mediana } \\
\text { (mín./máx.) }\end{array}$ & $\begin{array}{c}45 \\
(23 / 52)\end{array}$ & $\begin{array}{c}42 \\
(23 / 51)\end{array}$ & $\begin{array}{c}42 \\
(41 / 52)\end{array}$ & 0,064 \\
\hline \multicolumn{5}{|c|}{$\begin{array}{l}\text { Colesterol total } \\
(\mathrm{mg} / \mathrm{dL})\end{array}$} \\
\hline$M D \pm D P$ & $211 \pm 67$ & $188 \pm 41$ & $250 \pm 84$ & \\
\hline $\begin{array}{l}\text { Mediana } \\
\text { (mín./máx.) }\end{array}$ & $\begin{array}{c}191 \\
(110 / 371)\end{array}$ & $\begin{array}{c}188 \\
(110 / 295)\end{array}$ & $\begin{array}{c}234 \\
(134 / 371)\end{array}$ & 0,055 \\
\hline \multicolumn{5}{|c|}{ HDL-c (mg/dL) } \\
\hline$M D \pm D P$ & $51 \pm 15$ & $54 \pm 15$ & $45 \pm 12$ & \\
\hline $\begin{array}{l}\text { Mediana } \\
\text { (mín./máx.) }\end{array}$ & $\begin{array}{c}51 \\
(27 / 80)\end{array}$ & $\begin{array}{c}52 \\
(30 / 80)\end{array}$ & $\begin{array}{c}49 \\
(27 / 60)\end{array}$ & 0,261 \\
\hline \multicolumn{5}{|c|}{ LDL-c (mg/dL) } \\
\hline$M D \pm D P$ & $120 \pm 67$ & $95 \pm 43$ & $160 \pm 82$ & \\
\hline $\begin{array}{l}\text { Mediana } \\
\text { (mín./máx.) }\end{array}$ & $\begin{array}{c}111 \\
(29 / 281)\end{array}$ & $\begin{array}{c}106 \\
(29 / 176)\end{array}$ & $\begin{array}{c}166 \\
(46 / 281)\end{array}$ & 0,820 \\
\hline \multicolumn{5}{|c|}{ VLDL-c (mg/dL) } \\
\hline$M D \pm D P$ & $41 \pm 21$ & $39 \pm 17$ & $45 \pm 28$ & \\
\hline $\begin{array}{l}\text { Mediana } \\
\text { (mín./máx.) }\end{array}$ & $\begin{array}{c}36 \\
(13 / 88)\end{array}$ & $\begin{array}{c}37 \\
(13 / 67)\end{array}$ & $\begin{array}{c}29 \\
(21 / 88)\end{array}$ & 0,885 \\
\hline
\end{tabular}

Triglicérides $(\mathrm{mg} / \mathrm{dL})$

$\begin{array}{lcccc}\text { MD } \pm \text { DP } & 141 \pm 81 & 113 \pm 65 & 186 \pm 88 & \\ \text { Mediana } & 118 & 94 & 136 & 0,043^{*} \\ \text { (mín./máx.) } & (35 / 304) & (35 / 250) & (107 / 304) & \end{array}$

$\begin{array}{lcccc}\text { Castelli I } & & & & \\ \text { MD } \pm \text { DP } & 4,4 \pm 1,5 & 3,7 \pm 1,4 & 5,5 \pm 1,0 & \\ \text { Mediana } & 4,2 & 3,5 & 5,6 & 0,008^{*} \\ \text { (mín./máx.) } & (2,4 / 7,0) & (2,4 / 7,0) & (3,9 / 7,0) & \end{array}$

\section{Castelli II}

$\begin{array}{lcccc}M D \pm D P & 2,6 \pm 1,5 & 2,0 \pm 1,3 & 3,5 \pm 1,5 & \\ \text { Mediana } & 2,2 & 1,9 & 3,8 & 0,020 \text { * } \\ \text { (mín./máx.) } & (0,7 / 5,2) & (0,7 / 4,8) & (1,0 / 5,2) & \end{array}$

\begin{tabular}{lcccc} 
IMC (kg/m $\left.\mathbf{m}^{2}\right)$ & & & & \\
MD \pm DP & $29 \pm 6$ & $29 \pm 6$ & $30 \pm 7$ & \\
Mediana & 30 & 26 & 30 & 0,828 \\
(mín./máx.) & $(19 / 39)$ & $(20 / 38)$ & $(19 / 39)$ & \\
\hline
\end{tabular}

Teste de Mann-Whitney $\quad{ }^{*} \mathrm{p}<0,05$ (significante)

Castelli I - CT/HDL-c (índice); Castelli II - LDL-c/HDL-c (índice); CATE-ANL Cateterismo cardíaco anormal; CATE-NL - Cateterismo cardíaco normal; IMC - Índice de massa corpórea; $M D \pm D P$ - Média \pm desvio-padrão; Mín./máx. - Valores mínimo e máximo; P - Nível descritivo 
TABELA 3

MEDidAs de TENDÊNCIA CENTRAL E DE DISPERSÃo DOS FATORES DE RISCO RELACIONADOS AO LES DAS 21 PACIENTES SUBMETIDAS À CINEANGIOCORONARIOGRAFIA

\begin{tabular}{|c|c|c|c|c|}
\hline Variáveis & $\begin{array}{c}21 \\
\text { pacientes }\end{array}$ & $\begin{array}{c}13 \\
\text { CATE-NL }\end{array}$ & $\begin{array}{c}8 \\
\text { CATE-ANL }\end{array}$ & $\mathbf{P}$ \\
\hline \multicolumn{5}{|l|}{$\begin{array}{l}\text { Tempo de LES } \\
\text { (meses) }\end{array}$} \\
\hline $\mathrm{MD} \pm \mathrm{DP}$ & $132 \pm 66$ & $125 \pm 64$ & $142 \pm 74$ & \multirow[b]{2}{*}{0,664} \\
\hline $\begin{array}{l}\text { Mediana } \\
\text { (mín./máx.) }\end{array}$ & $\begin{array}{c}120 \\
(61 / 312)\end{array}$ & $\begin{array}{c}127 \\
(61 / 302)\end{array}$ & $\begin{array}{c}120 \\
(62 / 312)\end{array}$ & \\
\hline \multicolumn{5}{|l|}{$\begin{array}{l}\text { Idade início LES } \\
\text { (anos) }\end{array}$} \\
\hline$M D \pm D P$ & $31 \pm 10$ & $29 \pm 10$ & $35 \pm 8$ & \multirow[b]{2}{*}{0,128} \\
\hline $\begin{array}{l}\text { Mediana } \\
\text { (mín./máx.) }\end{array}$ & $\begin{array}{c}33 \\
(13 / 45)\end{array}$ & $\begin{array}{c}27 \\
(13 / 45)\end{array}$ & $\begin{array}{c}34 \\
(18 / 44)\end{array}$ & \\
\hline \multicolumn{5}{|l|}{$\mathrm{N}$ critérios ACR } \\
\hline $\begin{array}{l}\text { Mediana } \\
\text { (mín./máx.) }\end{array}$ & $\begin{array}{c}7 \\
(4 / 10)\end{array}$ & $\begin{array}{c}6 \\
(4 / 9)\end{array}$ & $\begin{array}{c}8 \\
(6 / 10)\end{array}$ & $0,018^{*}$ \\
\hline \multicolumn{5}{|l|}{ N SLE-DAI } \\
\hline$M D \pm D P$ & $6 \pm 5$ & $4 \pm 3$ & $11 \pm 3$ & \multirow[b]{2}{*}{$0,010^{*}$} \\
\hline $\begin{array}{l}\text { Mediana } \\
\text { (mín./máx.) }\end{array}$ & $\begin{array}{c}6 \\
(0 / 18)\end{array}$ & $\begin{array}{c}4 \\
(0 / 12)\end{array}$ & $\begin{array}{c}10 \\
(6 / 18)\end{array}$ & \\
\hline \multicolumn{5}{|l|}{ N SLICC/ACR-DI } \\
\hline$M D \pm D P$ & $2 \pm 2$ & $1 \pm 1$ & $4 \pm 1$ & \multirow[b]{2}{*}{0,003 * } \\
\hline $\begin{array}{l}\text { Mediana } \\
\text { (mín./máx.) }\end{array}$ & $\begin{array}{c}2 \\
(0 / 6)\end{array}$ & $\begin{array}{c}1 \\
(0 / 5)\end{array}$ & $\begin{array}{c}3 \\
(2 / 6)\end{array}$ & \\
\hline \multicolumn{5}{|c|}{${ }^{*} \mathrm{p}<0,05$ (significante) } \\
\hline $\begin{array}{l}\text { CATE-ANL - Cateterismo } \\
\text { MD } \pm \text { DP - Média } \pm \text { desvio } \\
\text { Lupus Erythematosus Dise } \\
\text { Collaborating Clinics/Ame }\end{array}$ & Activity Index; & CATE-NL - Ca & $\begin{array}{l}\text { temic Lupus } \\
\text { ge Index }\end{array}$ & $\begin{array}{l}\text { onormal; } \\
\text { Systemic } \\
\text { ernational }\end{array}$ \\
\hline
\end{tabular}

\section{DISCUSSÃO}

Os eventos cardiovasculares decorrentes de aterosclerose prematura têm sido reconhecidos como importantes fatores contribuintes para a morbidade e a mortalidade em pacientes com LES. No curso da doença, complexas interações entre o LES per se, a terapêutica e os fatores de risco tradicionais para DAC contribuem para a injúria vascular ${ }^{(7-9,14,36-39)}$. Os conhecimentos atuais a respeito da doença justificam conduta mais agressiva no controle dos fatores de risco clássicos e daqueles relacionados ao LES, que são preditivos de $\mathrm{DAC}^{(6,40-42)}$.

O tempo de uso e a dose cumulativa aproximada de prednisona foram calculados apenas para as pacientes com prontuários mais completos em seguimento clínico contínuo no nosso serviço desde o diagnóstico de LES. A seleção de apenas 48 das 90 pacientes para a avaliação da corti- coterapia teve a finalidade de minimizar os erros inerentes a esses cálculos por meio da revisão dos prontuários médicos. Considerando-se os subgrupos das pacientes com resultados normal e anormal de cintilografia miocárdica, não houve diferença significante com relação ao tempo de uso e à dose cumulativa de prednisona.

A alta dose de corticosteróide foi citada como fator de pior prognóstico na análise da sobrevida de pacientes com $\mathrm{LES}^{(43)}$. A corticoterapia prolongada e a maior dose cumulativa de prednisona foram associadas à presença da placa aterosclerótica em artérias carótidas ${ }^{(44)}$. Por outro lado, a maior duração da terapia com prednisona poderia representar um subgrupo de pacientes com doença mais grave ${ }^{(45)}$. Rahman, Gladman \& Urowitz ${ }^{(46)}$ referiram que a incidência de DAC prematura em pacientes com LES, mesmo na ausência de corticosteróide, foi maior $(4,5 / 1.000)$ que a observada pelo Framingham Heart Study $(2,8 / 1.000$ mulheres).

$\mathrm{Na}$ literatura, os antimaláricos têm sido apontados como poupadores de corticosteróides ${ }^{(47)}$ e capazes de reduzir os efeitos deletérios da corticoterapia no metabolismo lipídico $^{(48-50)}$. Este efeito poderia, hipoteticamente, diminuir o risco de aterogênese e conseqüentemente reduzir as alterações da cintilografia cardíaca. A análise das 48 pacientes com LES mostrou não haver diferença significante com relação ao tempo de uso de antimaláricos entre os subgrupos com e sem anormalidades de perfusão miocárdica.

Vasculite cutânea é a forma mais comum de vasculite em LES, ocorrendo em $20 \%$ a $50 \%$ dos pacientes. O seu reconhecimento pode estar correlacionado com a gravidade da doença e o envolvimento orgânico ${ }^{(51)}$. Vasculite foi interpretada como fator de pior prognóstico na análise de sobrevida de pacientes com $\operatorname{LES}^{(43,52)}$. No modelo de regressão logística proposto neste estudo, vasculite atual foi o fator de risco associado à alteração de perfusão miocárdica, sugerindo que a inflamação vascular possa ocorrer em outros territórios, incluindo a microcirculação coronária. Controladas as demais variáveis, o risco de pacientes com vasculite apresentarem anormalidades cintilográficas foi 23,5 vezes maior que o de pacientes sem vasculite no momento do exame.

Maior tempo de diagnóstico da doença e idade mais avançada na época do diagnóstico, mencionados como fatores de risco coronário relacionados ao LES por outros autores $^{(8,9,14)}$, não foram associados às anormalidades cintilográficas em nosso trabalho, mas devemos lembrar que participaram do estudo apenas pacientes com mais de cinco anos de doença. 
As anormalidades da cintilografia miocárdica nem sempre são confirmadas pelos achados da cineangiocoronariografia. A discrepância desses resultados pode ser imputada aos fatores inerentes ou relacionados às modalidades destes exames. A cintilografia miocárdica detecta alterações da perfusão cardíaca e a cineangiocoronariografia estuda a anatomia dos vasos coronários.

Cintilografia miocárdica anormal na ausência de placa aterosclerótica poderia ocorrer por prolapso de valva mitral, ponte miocárdica, doença de Chagas, estenose aórtica, bloqueio de ramo esquerdo, síndrome de Wolff-ParkinsonWhite, artefatos (interposição de mama e diafragma), ápice adelgaçado ou condições técnicas inadequadas, caracterizando os resultados falsos-positivos da cintilografia cardíaca ${ }^{(20,53)}$. Cintilografia miocárdica anormal com cineangiocoronariografia normal também poderia ocorrer nos casos em que a angiografia não identifica lesões ateroscleróticas por projeções inadequadas, superposição diafragmática, anomalias congênitas ou diferenças inter e intra-observadores, sendo estes resultados os falso-negativos da cineangiocoronariografia ${ }^{(20)}$.

Este foi o primeiro estudo da literatura com cineangiocoronariografia para a avaliação de pacientes com LES e anormalidades de perfusão cardíaca. Embora com relativa limitação pelo número reduzido de pacientes que se submeteram ao cateterismo cardíaco, nosso estudo mostrou que $38 \%$ das pacientes com anormalidades cintilográficas submetidas à cineangiocoronariografia apresentaram placas ateroscleróticas detectáveis em artérias coronárias. O subgrupo das pacientes com alterações coronárias apresentou maior número de fatores de risco tradicionais para DAC e maiores escores de critérios do ACR, de atividade (SLEDAI) e de danos permanentes (SLICC/ACR-DI) quando comparado ao subgrupo das pacientes com artérias coronárias normais. HAS, menopausa e hipertrigliceridemia foram os fatores de risco clássicos associados às lesões coronárias.

Sessenta e dois por cento das pacientes com alterações de perfusão miocárdica não apresentaram lesões coronárias

\section{REFERÊNCIAS}

1. Manzi S, Wasko MCM: Inflammation-mediated rheumatic diseases and atherosclerosis. Ann Rheum Dis 59: 321-25, 2000.

2. Libby P: Molecular bases of the acute coronary syndromes. Circulation 91: 2844-50, 1995.

3. Wick G, Schett G, Amberger A, Kleindienst R, Xu Q: Is atherosclerosis an immunologically mediated disease? Immunol Today 16: 27-33, 1995. detectáveis em estudo invasivo. Há indícios de que os defeitos de perfusão miocárdica dessas pacientes representem disfunção endotelial, sugerindo que as anormalidades cintilográficas sejam decorrentes de um estágio subclínico de DAC em LES $^{(54)}$.

As pacientes com alterações cintilográficas e lesões coronárias ateroscleróticas estão sendo acompanhadas nos ambulatórios das Disciplinas de Reumatologia e de Cardiologia da Instituição. No seguimento clínico, uma paciente teve IAM extenso em parede anterior de ventrículo esquerdo, complicado por acidente vascular cerebral (AVC) com evolução fatal. Três pacientes foram submetidas à angioplastia com colocação de stent para a artéria coronária direita (um caso) e para a artéria descendente anterior (dois casos). Uma paciente submeteu-se à cirurgia de revascularização miocárdica e três pacientes estão recebendo tratamento medicamentoso.

Ressaltamos em nosso estudo a importância da vasculite como fator de risco determinante do estudo de perfusão miocárdica e dos clássicos fatores de risco coronário associados à lesão de artérias coronárias. Salientamos, ainda, a necessidade do reconhecimento e efetivo controle de todos os fatores de risco coronário (tradicionais ou relacionados ao LES) e da investigação clínica de doença aterosclerótica como diagnóstico diferencial de dor torácica em mulheres com LES, mesmo em fase de pré-menopausa.

Nossos dados sugerem que as pacientes com LES devam ter os fatores de risco controlados de forma contundente e aquelas com doença há mais de cinco anos e presença de, pelo menos, quatro fatores de risco tradicionais sejam avaliadas com o estudo de perfusão cardíaca, mesmo na ausência de sintomas de insuficiência coronária. Em caso de maior risco para doença cardiovascular, a cintilografia miocárdica pode ser usada como rastreamento de DAC em LES. As pacientes com anormalidades cintilográficas e quatro ou mais fatores de risco clássicos para DAC sofrem alto risco de apresentar estenoses de artérias coronárias na cineangiocoronariografia e poderão se beneficiar de tratamento mais agressivo.

4. Kullo IJ, Edwards WD, Schwartz RS: Vulnerable plaque pathobiology and clinical implications. Ann Intern Med 129: 105060, 1998.

5. George J, Afek A, Gilburd B, Harats D, Shoenfeld Y: Autoimmunity in atherosclerosis - lessons from experimental models. Lupus 9: 223-7, 2000.

6. Manzi S, Kuller LH, Edmundowicz D, Sutton-Tyrrell K: Vascular imaging - changing the face of cardiovascular research. Lupus 9: 176-182, 2000. 
7. Gladman DD, Urowitz MB: Morbidity in systemic lupus erythematosus. J Rheumatol 14(suppl 13): 223-6, 1987.

8. Petri M, Perez-Gutthann S, Spence D, Hochberg MC: Risk factors for coronary artery disease in patients with systemic lupus erythematosus. Am J Med 93: 513-9, 1992.

9. Manzi S, Meilahn EN, Rairie JE, et al: Age-specific incidence rates of myocardial infarction and angina in women with systemic lupus erythematosus - comparison with the Framingham study. Am J Epidemiol 145: 408-15, 1997.

10. Bruce IN, Gladman DD, Urowitz MB: Detection and modification of risk factors for coronary artery disease in patients with systemic lupus erythematosus - a quality improvement study. Clin Exp Rheumatol 16: 435-40, 1998.

11. Bruce IN, Urowitz MB, Gladman DD, Hallett DC: Natural history of hypercholesterolemia in systemic lupus erythematosus. J Rheumatol 26: 2137-43, 1999.

12. Bruce IN, Urowitz MB, Ibanez D, Steiner G, Gladman DD: The prevalence of "Framingham risk factors" for coronary artery disease in women with SLE - a cohort control study. Arthritis Rheum 43(suppl): S246, abstract 1080, 2000.

13. Sella EMC, Sato EI: Estudo de perfusão miocárdica e fatores de risco coronário em pacientes com lúpus eritematoso sistêmico. Rev Bras Reumatol 43: 73-83, 2003.

14. Jonsson H, Nived O, Sturfelt G: Outcome in systemic lupus erythematosus - a prospective study of patients from a defined population. Medicine 68: 141-50, 1989.

15. Hosenpud JD, Montanaro A, Hart MV, et al: Myocardial perfusion abnormalities in asymptomatic patients with systemic lupus erythematosus. Am J Med 77: 286-92, 1984.

16. Gazarian M, Feldman BM, Benson LN, Gilday DL, Laxer RM, Silverman ED: Assessment of myocardial perfusion and function in childhood systemic lupus erythematosus. J Pediatr 132: 109-16, 1998.

17. Schillaci $\mathrm{O}$, Laganà $\mathrm{B}$, Danieli $\mathrm{R}$, et al: Technetium-99m sestamibi single-photon emission tomography detects subclinical myocardial perfusion abnormalities in patients with systemic lupus erythematosus. Eur J Nucl Med 26: 713-7, 1999.

18. Bruce IN, Burns RJ, Gladman DD, Urowitz MB: Single photon emission computed tomography dual isotope myocardial perfusion imaging in women with systemic lupus erythematosus $-I$. Prevalence and distribution of abnormalities. J Rheumatol 27: 2372-7, 2000.

19. Sun SS, Shiau YC, Tsai SC, Lin CC, Kao A, Lee CC: The role of Technetium-99m sestamibi myocardial perfusion single-photon emission computed tomography (SPECT) in the detection of cardiovascular involvement in systemic lupus erythematosus patients with non-specific chest complaints. Rheumatology 40: 1106-11, 2001.

20. Meneghelo RS: Teste ergométrico, cintilografia e cinecoronariografia - correlação e discrepâncias. Rev Soc Cardiol Estado de São Paulo 5: 285-9, 1995 .

21. Tan EM, Cohen AS, Fries JF, et al: The 1982 revised criteria for the classification of systemic lupus erythematosus. Arthritis Rheum 25: 1271-7, 1982

22. Hochberg MC: Updating the American College of Rheumatology revised criteria for the classification of systemic lupus erythematosus. Arthritis Rheum 40: 1725, 1997 (letter).

23. Departamento de Ergometria e Reabilitação Cardiovascular da Sociedade Brasileira de Cardiologia: Consenso Nacional de Ergometria. Arq Bras Cardiol 65: 189-211, 1995.
24. Bombardier C, Gladman DD, Urowitz MB, Caron D, Chang CH, Committee on Prognosis Studies in SLE: Derivation of the SLEDAI - a disease activity index for lupus patients. Arthritis Rheum 35: 630-40, 1992.

25. Gladman D, Ginzler E, Goldsmith C, et al: The development and initial validation of the systemic lupus international collaborating clinics/American College of Rheumatology damage index for systemic lupus erythematosus. Arthritis Rheum 39: 363-69, 1996.

26. Gladman DD, Urowitz MB, Goldsmith $\mathrm{CH}$, et al: The reliability of the systemic lupus international collaborating clinics/American College of Rheumatology damage index in patients with systemic lupus erythematosus. Arthritis Rheum 40: 809-13, 1997.

27. Sociedade Brasileira de Cardiologia: Segundo Consenso Brasileiro sobre dislipidemias - avaliação, detecção e tratamento. Arq Bras Cardiol 67: 109-28, 1996.

28. European Society of Cardiology, European Atherosclerosis Society, European Society of Hypertension, International Society of Behavioural Medicine, European Society of General Practice/Family Medicine, European Heart Network: Prevention of coronary heart disease in clinical practice - recommendations of the second Joint Task Force of European and other Societies on coronary prevention. Eur Heart J 19: 1434-503, 1998.

29. Expert Panel on Detection, Evaluation, and Treatment of High Blood Cholesterol in Adults: Summary of the second report of the National Cholesterol Education Program expert panel on detection, evaluation, and treatment of high blood cholesterol in adults (adult treatment panel II). JAMA 269: 3015-23, 1993.

30. Expert Panel on Detection, Evaluation, and Treatment of High Blood Cholesterol in Adults: National Cholesterol Education Program second report of the expert panel on detection, evaluation, and treatment of high blood cholesterol in adults (adult treatment panel II). Circulation 89: 1329-445, 1994.

31. American College of Cardiology/American Heart Association Task Force on Practice Guidelines (Committee on Management of Patients with Chronic Stable Angina): ACC/AHA/ACP-ASIM guidelines for the management of patients with chronic stable angina: executive summary and recommendations. Circulation 99: 2829-48, 1999.

32. American College of Cardiology/American Heart Association Task Force on Assessment of Diagnostic and Therapeutic Cardiovascular Procedures (Committee on Radionuclide Imaging)/American Society of Nuclear Cardiology: Guidelines for clinical use of cardiac radionuclide imaging. J Am Coll Cardiol 25: 521-47, 1995.

33. American College of Cardiology/American Heart Association Task Force on Practice Guidelines (Committee on Exercise Testing): ACC/AHA guidelines for exercise testing. J Am Coll Cardiol 30: 260-315, 1997.

34. Siegel J: Estatística não-paramétrica, Brasil, McGraw Hill do Brasil, p. 350,1981

35. Kelsey JL, Hwittemore AS, Evans AS, Thompson WD: Methods in observational epidemiology, 2nd ed., Oxford, University Press, p.432, 1996.

36. Ginzler E, Berg A: Mortality in systemic lupus erythematosus. J Rheumatol 14(suppl13): 218-22, 1987.

37. Urowitz MB, Gladman DD: Evolving spectrum of mortality and morbidity in SLE. Lupus 8: 253-5, 1999.

38. Ward MM: Premature morbidity from cardiovascular and cerebrovascular diseases in women with systemic lupus erythematosus. Arthritis Rheum 42: 338-46, 1999. 
39. Esdaile JM, Abrahamowicz M, Grodzicky T, et al: Traditional Framingham risk factors fail to fully account for accelerated atherosclerosis in systemic lupus erythematosus. Arthritis Rheum 44: 2331-7, 2001.

40. Aranow C, Ginzler EM: Epidemiology of cardiovascular disease in systemic lupus erythematosus. Lupus 9: 166-9, 2000.

41. Bruce IN, Gladman DD, Urowitz MB: Systemic lupus erythematosus - premature atherosclerosis in systemic lupus erythematosus. Rheum Dis Clin North America 26: 257-78, 2000.

42. Al-Herz A, Ensworth S, Shojania K, Esdaile JM: Cardiovascular risk factor screening in systemic lupus erythematosus. J Rheumatol 30: 493-6, 2003.

43. Massardo L, Martínez ME, Jacobelli S, Villarroel L, Rosenberg H, Rivero S: Survival of chilean patients with systemic lupus erythematosus. Semin Arthritis Rheum 24: 1-11, 1994.

44. Manzi S, Selzer F, Sutton-Tyrrell K, et al: Prevalence and risk factors of carotid plaque in women with systemic lupus erythematosus. Arthritis Rheum 42: 51-60, 1999.

45. Petri M: Detection of coronary artery disease and the role of traditional risk factors in the Hopkins lupus cohort. Lupus 9: 170-5, 2000.

46. Rahman P, Gladman DD, Urowitz MB: Premature coronary artery disease in systemic lupus erythematosus in the absence of corticosteroid use. J Rheumatol 27: 1323-5, 2000 (letter).

47. Meinão IM, Sato EI, Andrade LEC, Ferraz MB, Atra E: Controlled trial with chlroquine diphosphate in systemic lupus erythematosus. Lupus 5: 237-41, 1996.
48. Hodis HN, Quismorio Jr FP, Wickham E, Blankenhorn DH: The lipid, lipoprotein, and apolipoprotein effects of hydroxychloroquine in patients with systemic lupus erythematosus. J Rheumatol 20: 661-5, 1993.

49. Petri M, Lakatta C, Magder L, Goldman D: Effect of prednisone and hydroxychloroquine on coronary artery disease risk factors in systemic lupus erythematosus - a longitudinal data analysis. Am J Med 96: 254-9, 1994.

50. Rahman P, Gladman DD, Urowitz MB, Yuen K, Hallett D, Bruce IN: The cholesterol lowering effect of antimalarial drugs is enhanced in patients with lupus taking corticosteroid drugs. J Rheumatol 26: 325-30, 1999.

51. Fessler BJ, Hoffman GS: Systemic lupus erythematosus and the cardiovascular system - vasculitis. In: Lahita RG (ed.). Systemic lupus erythematosus. 3rd ed., San Diego, Academic Press, section V, chapter 33, pp. 707-17, 1999.

52. Sato EI, Vieira ALS, Pochini AC, et al: Fatores prognósticos no lúpus eritematoso sistêmico. Rev Bras Reumatol 35: 235-41, 1995.

53. Martins LRF: Interpretação das imagens de perfusão do miocárdio. Rev Soc Cardiol Estado de São Paulo 5: 262-70, 1995.

54. Sato EI, Sella EMC, Lima DSN: Myocardial perfusion scintigraphy and endothelial function in systemic lupus erythematosus patients. Arthritis Rheum 46(suppl): S53, abstract 26, 2002. 